Geometry 8 Topology

Volume 9 (2005) 2303-2317

Published: 10 December 2005

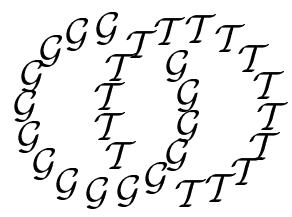

\title{
Universal manifold pairings and positivity
}

Michael H Freedman $^{1}$, Alexei Kitaev ${ }^{2}$, Chetan NayaK ${ }^{1,3}$

Johannes K Slingerland ${ }^{1}$, Kevin Walker ${ }^{1}$ and Zhenghan Wang ${ }^{4}$

1: Microsoft Research, 1 Microsoft Way, Redmond, WA 98052, USA

2: California Institute of Technology, Pasadena, CA 91125, USA

3: Department of Physics and Astronomy, UCLA, CA 90095-1547, USA

4: Dept of Mathematics, Indiana University, Bloomington, IN 47405, USA

michaelf@microsoft.com, kitaev@iqi.caltech.edu, nayak@physics.ucla.edu

joost@microsoft.com, kwalker@microsoft.com, zhewang@indiana.edu

\begin{abstract}
Gluing two manifolds $M_{1}$ and $M_{2}$ with a common boundary $S$ yields a closed manifold $M$. Extending to formal linear combinations $x=\Sigma a_{i} M_{i}$ yields a sesquilinear pairing $p=\langle$,$\rangle with values in (formal linear combinations of) closed manifolds. Topological$ quantum field theory (TQFT) represents this universal pairing $p$ onto a finite dimensional quotient pairing $q$ with values in $\mathbb{C}$ which in physically motivated cases is positive definite. To see if such a "unitary" TQFT can potentially detect any nontrivial $x$, we ask if $\langle x, x\rangle \neq 0$ whenever $x \neq 0$. If this is the case, we call the pairing $p$ positive. The question arises for each dimension $d=0,1,2, \ldots$. We find $p(d)$ positive for $d=0,1$, and 2 and not positive for $d=4$. We conjecture that $p(3)$ is also positive. Similar questions may be phrased for (manifold, submanifold) pairs and manifolds with other additional structure. The results in dimension 4 imply that unitary TQFTs cannot distinguish homotopy equivalent simply connected 4-manifolds, nor can they distinguish smoothly $s$-cobordant 4 -manifolds. This may illuminate the difficulties that have been met by several authors in their attempts to formulate unitary TQFTs for $d=3+1$. There is a further physical implication of this paper. Whereas 3-dimensional ChernSimons theory appears to be well-encoded within 2-dimensional quantum physics, e.g. in the fractional quantum Hall effect, Donaldson-Seiberg-Witten theory cannot be captured by a 3 -dimensional quantum system. The positivity of the physical Hilbert spaces means they cannot see null vectors of the universal pairing; such vectors must map to zero.
\end{abstract}

AMS Classification numbers Primary: 57R56, 53D45

Secondary: 57R80, 57N05, 57N10, 57N12, 57N13

Keywords: Manifold pairing, unitary, positivity, TQFT, s-cobordism

Proposed: Robion Kirby

Seconded: Peter Teichner, Cameron Gordon

Received: 25 May 2005

Revised: 2 December 2005 


\section{Introduction}

We begin by establishing notation. We will work with oriented, compact, possibly disconnected, smooth manifolds, although some comments will also be made concerning the unoriented case. The choice of smooth category might be essential: Our vector $x$ is constructed from a counterexample to the $s$-cobordism theorem, which is still open in the topological category.

Let $S$ be a $d-1$ dimensional manifold and let $\mathcal{M}_{S}$ be the $\mathbb{C}$-vector space of (finite) formal combinations of manifolds $M_{i}$ with $\partial M_{i}=S$, so $x=\sum_{i} a_{i} M_{i} \in$ $\mathcal{M}_{S}$. (Note: If $S$ does not bound, dimension $\left(\mathcal{M}_{S}\right)=0$.) If we denote $S$ with the opposite orientation by $\bar{S}$, then we have a bilinear pairing

$$
\mathcal{M}_{S} \times \mathcal{M}_{\bar{S}} \longrightarrow \mathcal{M}
$$

given by $\left(\sum_{i} a_{i} M_{i}, \sum_{j} b_{j} N_{j}\right) \longrightarrow \sum_{i, j} a_{i} b_{j} M_{i} \cup_{S} N_{j}$, where $\mathcal{M}=\mathcal{M}_{\emptyset}$ is the vector space of formal linear combinations of closed $d$-manifolds. To fit better with the role of Hilbert space in physics we choose to rewrite (11) as a sesquilinear pairing

$$
\langle,\rangle: \mathcal{M}_{S} \times \mathcal{M}_{S} \longrightarrow \mathcal{M},\left\langle\sum_{i} a_{i} M_{i}, \sum_{j} b_{j} N_{j}\right\rangle=\sum_{i, j} a_{i} \bar{b}_{j} M_{i} \cup_{S} \bar{N}_{j}
$$

which is linear in the first entry and conjugate linear in the second. The map from $\mathcal{M}_{S} \times \mathcal{M}_{\bar{S}}$ to $\mathcal{M}_{S} \times \mathcal{M}_{S}$ which intertwines between the pairings is just the conjugate linear extension of orientation reversal on the second factor.

We need to be perfectly clear about when two boundary manifolds $M_{i}$ and $M_{j}$ are considered the same element of $\mathcal{M}_{S}$. A basis element $M_{i}$ of $\mathcal{M}_{S}$ is a manifold $M_{i}$ together with a diffeomorphism $f_{i}$ of $\partial M_{i}$ to $S$. We say $\left(M_{i}, f_{i}\right)$ and $\left(M_{j}, f_{j}\right)$ are equivalent if there is a diffeomorphism $\phi: M_{i} \longrightarrow M_{j}$ such that:

$$
\left.f_{j} \circ \phi\right|_{\partial M_{i}}=f_{i} .
$$

With this definition, we have examples where the manifolds $M_{i}, M_{j}$ are the same, but attached differently to the boundary and hence not equivalent. Perhaps the simplest of these is shown in Figure 1 where the manifolds both consist of two line segments, attached to the four boundary points in different ways. Less trivially, a surface bounds infinitely many distinct handle bodies parameterized by the cosets: $M C_{g} / H C_{g}$; the genus $=g$ mapping class group modulo the subgroup which extends over a fixed handlebody.

Occasionally we consider simply the set of bounded manifolds up to equivalence

(ie, the basis vectors of $\mathcal{M}_{S}$ ) and denote this set by $\dot{\mathcal{M}}_{S}$. We reserve the dot to mean "unlinearize". 

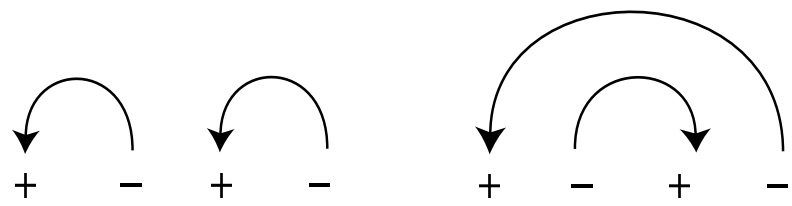

Figure 1: Two inequivalent examples of 1-manifolds with boundary. In both cases, the manifold consists of two oriented line segments and the boundary of two positively and two negatively oriented points, but the attaching maps are different

Our definitions easily extend to (manifold, submanifold) pairs (if $K \subset M$ is a submanifold we always assume $\partial K \subset \partial M)$. Let $\mathcal{M}^{d, k}$ be the space of formal combinations of $(d$-manifold, $k$-submanifold $)$ pairs. If $(S, L)$ is a fixed $\left((d-1)\right.$-manifold, $(k-1)$-submanifold) pair, we may define $\mathcal{M}_{(S, L)}^{d, k}$ to be formal combinations of bounding ( $d$-manifold, $k$-submanifold) pairs with an equivalence relation analogous to (3) and a sesquilinear pairing:

$$
\mathcal{M}_{S, L}^{d, k} \times \mathcal{M}_{S, L}^{d, k} \longrightarrow \mathcal{M}^{d, k}
$$

by a formula like (2).

A variant on gluing pairs is to require the outer manifolds to be as simple as possible, spheres and disks. This gives sesquilinear "tangle pairings":

$$
\Im_{L}^{d, k} \times \Im_{L}^{d, k} \longrightarrow \mathcal{L}^{d, k}
$$

where $L$ is a fixed $(k-1)$-submanifold of $S^{d-1}$ and $\Im_{L}^{d, k}$ is the span of $k$-submanifolds in $D^{d}$ bounded by $L$. The target $\mathcal{L}^{d, k}$ is the span of $k^{-}$ submanifolds in $S^{d}$.

For all the sesquilinear pairings above we may ask if they are positive, that is, whether $\langle x, x\rangle=0$ implies $x=0$. The motivation is to understand how much of manifold topology can potentially be detected by unitary topological quantum field theories (UTQFTs. See [1] for a definition). To touch on only the most elementary aspect of this structure, a UTQFT should assign a scalar to a closed $d$-manifold and a finite dimensional Hilbert space $V_{S}$ to each $(d-1)$ manifold $S$. For $X$ with $\partial X=S$, a vector $\widetilde{X} \in V_{S}$ is assigned and if $X^{\prime}$ also satisfies $\partial X^{\prime}=S$ then $\left\langle\widetilde{X}, \widetilde{X}^{\prime}\right\rangle$ must equal the closed manifold invariant of $X \bar{X}^{\prime}:=X \cup_{S} \bar{X}^{\prime}$. Clearly if one of our pairings is not formally positive, there will be an $x=\Sigma a_{i} X_{i} \neq 0$ for which $\langle x, x\rangle=0$, and no unitary TQFT will be able to distinguish the combination $x$ from zero. This question is (roughly) in the same spirit as asking if the Jones polynomial detects all knots.

To make the connection to TQFTs more exact one might choose to enhance our manifolds with framings, spin structure, $p_{1}$-structures, etc..., the necessary 
input for certain TQFTs. But the investigation is at such a preliminary stage that this level of detail is not yet warranted. Also, one may note that the invariants for closed manifolds often depend only weakly on the extra structures in the definition of TQFTs, so our results for closed manifolds may already be useful in such cases. With the definition complete, let us do what is easy.

\section{Lowest dimensions}

Theorem 2.1 The following pairings are positive:

$$
\mathcal{M}_{Y}^{d} \times \mathcal{M}_{Y}^{d} \longrightarrow \mathcal{M}^{d}
$$

for $d=0,1,2$,

$$
\mathcal{M}_{Y, L}^{d, k} \times \mathcal{M}_{Y, L}^{d, k} \longrightarrow \mathcal{M}^{d, k}
$$

for $d=0,1,2 ; k<d$ and

$$
\Im_{L}^{d, k} \times \Im_{L}^{d, k} \longrightarrow \mathcal{L}^{d, k}
$$

for $d=0,1,2 ; k<d$.

Conjecture 2.2 Theorem 2.1 extends to $d=3$ in the above cases.

Lemma 2.3 Suppose there exists a function (the "complexity function") $C: \dot{\mathcal{M}}^{d} \rightarrow \mathcal{V}$, where $\mathcal{V}$ is some partially ordered set, such that for all $M, N \in$ $\dot{\mathcal{M}}_{Y}^{d}, M \neq N$ implies $C(M \cup \bar{N})<\max (C(M \cup \bar{M}), C(N \cup \bar{N}))$. Then the pairing for $\mathcal{M}_{Y}^{d}$ is positive. Similar statements hold for $\mathcal{M}_{Y, L}^{d, k}$ and $\Im_{L}^{d, k}$.

Proof The hypothesis of the lemma implies that the terms of maximal complixity in the right-hand side of Equation 2 all lie on the diagonal. Since all coefficients on the diagonal are positive, there can be no cancellation among these terms.

Proof of Theorem 2.1 By the previous Lemma, it suffices in each case to define an appropriate complexity function $C$.

We ignore $\mathcal{L}^{d, k}$, since these cases are implied by the $\mathcal{M}^{d, k}$ cases.

For $\mathcal{M}^{1}$, define $C(M)$ to be the number of components of $M$. Let $Y$ be the 0 -manifold with $j$ positive and $j$ negative points. Let $M, N \in \mathcal{M}_{Y}^{1}$, and assume for the moment that neither $M$ nor $N$ contain closed components. Then $C(M \cup \bar{M})=C(N \cup \bar{N})=j$. If $M \neq N$ then at least one component 
of $M \cup \bar{N}$ contains 4 or more arcs, and so $C(M \cup \bar{N})<j$. Then general case (where $M$ and $N$ might have closed components) is similar.

Next consider $\mathcal{M}_{Y}^{2} \times \mathcal{M}_{Y}^{2} \longrightarrow \mathcal{M}^{2}$, where $Y$ is the disjoint union of $j$ circles. Let $M \in \dot{\mathcal{M}}^{2}$ (ie, $M$ is a closed oriented 2 -manifold). Let $n$ be the number of connected components of $M$, let $\chi$ be the Euler characteristic of $M$, and let $\chi_{1}, \ldots, \chi_{n}$ be the Euler characteristics of the components of $M$, listed in increasing order. Define the complexity of $M$ to be the lexicographic tuple

$$
C(M)=\left(n,-\chi,-\chi_{1}, \ldots,-\chi_{n}\right)
$$

The smallest integer that can appear in the tuple is -2 so we formally pad tuples by adding a list of -3 's at the end so that tuples of different lengths can be compared.

Now let $M, N \in \dot{\mathcal{M}}_{Y}^{2}$ and assume $M \neq N$. For simplicity, assume that neither $M$ nor $N$ contain any closed components. $M$ determines a partition of the components of $Y$ : two components of $Y$ are in the same part of the partition if they and connected by a component of $M$. In the same way $N$ also determines a partition of the components of $Y$. If these two partions differ then $M \cup$ $\bar{N}$ has fewer components than at least one of $M \cup \bar{M}$ and $N \cup \bar{N}$, so the hypothesis of the lemma is satisfied. We assume from now on that the partitions associated to $M$ and $N$ are the same. If $\chi(M)<\chi(N)$, then $C(M \cup \bar{N})<$ $C(M \cup \bar{M})$ (consider the second component of the complexity tuple), so we assume from now on that $\chi(M)=\chi(N)$. The components of $M$ and $N$ are paired according to their common partition of the components of $Y$. Since $M \neq N$, there must be at least one pair with differing Euler characteristics. Amongst such components in non-matching pairs, choose the one with lowest Euler characteristic, and assume WLOG that this extremal component belongs to $M$. It follow that $C(M \cup \bar{N})<C(M \cup \bar{M})$, and we are done.

The remaining cases of Theorem 2.1 are proved similarly. The most complicated case is $\mathcal{M}^{2,1}$. For $(M, K) \in \dot{\mathcal{M}}^{2,1}$ define $C(M, K)$ to be the lexicographic triple $(C(M), C(K), C(M \backslash K))$, where $C$ is as above for plain 1- and 2-manifolds.

Remark 2.4 Because the Turaev-Viro TQFTs do not require orientations it is reasonable to also investigate the universal pairings in the context of unoriented manifolds. In this context, define $\left.\left\langle\sum_{i} a_{i} M_{i}, \sum_{j} b_{j} N_{j}\right\rangle=\sum_{i, j} a_{i} \bar{b}_{j} M_{i} N_{j}\right\rangle$. Theorem 2.1] continues to hold in the unoriented context. 


\section{Three dimensional pairings}

This might be the most interesting case and we hope it will be the subject of future research. We establish positivity only in a few rather easy cases where all the work is contained in old theorems.

Theorem 3.1 The pairings $\mathcal{M}_{S}^{3} \times \mathcal{M}_{S}^{3} \longrightarrow \mathcal{M}^{3}, S$ a fixed (possibly empty) union of 2-spheres, and $\Im_{2}^{3,1} \times \Im_{2}^{3,1} \longrightarrow \mathcal{L}^{3,1}$, where 2 denotes two points with opposite orientations, are positive.

Proof The essential ingredient in both arguments is the existence and uniqueness of prime decompositions of 3 -manifolds [14] and knots [15]. Using this, both cases reduce to the following lemma:

Lemma 3.2 Consider the polynomial rings $\mathbb{C}\left[p_{i} \ldots p_{n}\right]$ on indeterminates $p_{1}, \ldots, p_{n}$ and with a fixed antilinear involution - which sends indeterminates to indeterminates. The natural sesquilinear pairing on these rings,

$$
\begin{array}{cll}
\mathbb{C}\left[p_{1} \ldots p_{n}\right] & \otimes \mathbb{C}\left[p_{1} \ldots p_{n}\right] & \longrightarrow \mathbb{C}\left[p_{1} \ldots p_{n}\right] \\
a & \otimes b & \mapsto
\end{array}
$$

is formally positive.

Proof We define the complexity of a monomial as some kind of list of prime powers it contains. If two distinct primes are related by involution we form a lexicographic pair: (sum of the two exponents, the smaller of the two exponents). For primes paired with themselves the pair is simply (exponent, zero). Note that the latter case will arise in the proof of theorem 3.1] since some prime knots (3-manifolds) are diffeomorphic to their arrow reversed (orientation reversed) mirror image.

Now list the pairs in order (padded by $(0,0)$ 's) and use this list of pairs to lexicographically order monomials. Suppose $X_{i}=\prod_{k} p_{i, k}^{d_{i, k}}$ are monomials, $x=\Sigma a_{i} X_{i}$, then the monomials of greatest complexity in $\langle x, x\rangle$ are among the diagonal terms $a_{i} \bar{a}_{i} X_{i} \bar{X}_{i}=a_{i} \bar{a}_{i} \prod_{k} p_{i, k}^{d_{i, k}} \bar{p}_{i, k}^{d_{i, k}}$. This is easily checked.

We make one further observation:

Theorem 3.3 Consider the pairing $\mathcal{M}_{S}^{3} \otimes \mathcal{M}_{S}^{3} \longrightarrow \mathcal{M}^{3}$ where $S$ is a connected genus $=g$ surface. Suppose $x$ is a linear combination $\Sigma a_{i} X_{i}$, where each $X_{i}$ is a handle body of genus $=g$ (but each attached to $S$ differently), then $\langle x, x\rangle=0$ implies each $a_{i}=0$. 
Proof The diagonal terms yield $\sum_{i} a_{i} \bar{a}_{i}\left(S^{1} \times S^{2} \# \cdots \# S^{1} \times S^{2}\right)$, a positive multiple of the double of the genus $=g$ handle body. Could an off diagonal term cancel this contribution? Such an off diagonal term must have $X_{i} \bar{X}_{j}$ diffeomorphic to $N_{g}:=\underset{g \text { copies }}{\#} S^{1} \times S^{2}$, and in fact constitute an exotic minimal Heegaard decomposition of $N_{g}$ (if not exotic, our equivalence relation on bounding manifolds (3) - allows us to write the $X_{i} \bar{X}_{j}$ term as an untwisted double). This contradicts a theorem of Waldhausen's [16] which shows that any two Heegaard decompositions of $N_{g}$ are related by an (orientation preserving) diffeomorphism.

Remark 3.4 3-manifold topology, in practice, is often two subjects, "compression-theory" and hyperbolic geometry, patched together. Some geometric arguments (about volumes, lengths, and Ricci flow [6]) offer hope for positivity on the hyperbolic side of the subject and Theorem 3.3 offers hope on the compression side. For these reasons we conjecture that the three dimensional pairings are positive.

\section{4-manifold pairings}

For a variety of 3 -manifolds $S$, we can exhibit vectors $x=M-M^{\prime} \in \mathcal{M}_{S}^{4}$ such that

$$
\langle x, x\rangle=M \bar{M}-M \bar{M}^{\prime}-M^{\prime} \bar{M}+M^{\prime} \bar{M}^{\prime}=0 \in \mathcal{M}^{4}=\mathcal{M}_{\emptyset}^{4} .
$$

In all cases the difference between $M$ and $M^{\prime}$ is a matter of differentiable structure on an underlying Poincaré pair (or, when $\pi_{1}(M)=\{e\}$, an underlying topological manifold). In one example of such an $x$, which has its roots in [3, 4, $M$ and $M^{\prime}$ are both copies of the "Mazur manifold" and $S=\partial M$, but $M$ is attached by the "identity" and $M^{\prime}$ is attached by a diffeomorphism $\theta$ of the boundary which does not extend to a diffeomorphism of the interior (but does extend as a homeomorphism). According to the definition in equation (3), $M$ and $M^{\prime}$ are distinct, so $x \neq 0$.

In 4. Akbulut and Kirby showed, by direct handle manipulation, that doubling Mazur's contractible manifold (via the identity on its integral homology sphere boundary) yields the smooth 4 -sphere, $M \bar{M} \cong S^{4}$ and remarkably, the $\theta-$ twisted double is also diffeomorphic to the 4-sphere, $M \bar{M}^{\prime}=S^{4}$ (since $\theta^{2}=$ id, $M^{\prime} \bar{M} \cong M \bar{M}^{\prime}$ and trivially $\left.M^{\prime} \bar{M}^{\prime} \cong M \bar{M}\right)$. In [9], one of us showed that $M$ and $M^{\prime}$ constituted the same topological manifold structure on the 
Poincaré pair. Then, with the introduction of gauge theory in topology, it became possible to distinguish $M$ and $M^{\prime}$ as smooth structures. Akbulut [3] did this by showing that the Kummer surface $K$ and one of its logarithmic transforms $K^{\prime}$, although distinguished by Donaldson invariants, differed on a combinatorial level only by cutting out an embedded $M$ and regluing it via $\theta$. This shows that $M \neq M^{\prime} \in \mathcal{M}_{S}^{4}$. In fact, if $C$ is the closed component $C=\overline{K \backslash M}$, we may write

$$
\begin{gathered}
K=C \cup M, \quad K^{\prime}=C \cup M^{\prime} \\
K \neq K^{\prime} \Longrightarrow M \neq M^{\prime} .
\end{gathered}
$$

SO

The pair of manifolds $K, K^{\prime}$ is but one of the many examples of pairs of (smoothly) $h$-cobordant but non-diffeomorphic manifolds.

Later, a comprehensive analysis of 1 -connected $h$-cobordisms extended Akbulut's result (see [8, 13, 12]). The following picture of the general 1-connected 5 -dimensional $h$-cobordism $(W ; P, Q)$ emerges. First, handles of indices $0,1,4$ and 5 are cancelled. Let $L \subset W$ be the middle level between the 2 -handles and 3 -handles. In $L$ lie the ascending 2 -spheres $A$ of the 2 -handles and the descending 2 -spheres $D$ of the 3 -handles. It is possible to engulf $A \cup D$ in a $4-$ manifold $N \subset L$, where $N$ is homotopy equivalent to a wedge of 2 -spheres. The gradient lines through $N$ define a sub- $h$-cobordism $\left(X ; M, M^{\prime}\right) \subset(W ; P, Q)$. Combinatorially, $X$ is obtained from $N \times[-1,1]$ by attaching 3 -handles to $D \times\{1\}$ and (up-side down) 3 -handles to $A \times\{-1\}$, so $M=N / A$ and $M^{\prime}=$ $N / D$, where / represents "surgery". ( $M$ and $M^{\prime}$ no longer denote the Mazur manifold, being instead generalizations.) The gradient lines constitue a product structure on the complementary $h$-cobordism $\left(\overline{W \backslash X} ; \overline{P \backslash M}, \overline{Q \backslash M^{\prime}}\right)$, so $\left(X ; M, M^{\prime}\right)$ is the "interesting" part. By choosing $N$ with care, the following conditions can be achieved:

(1) $X \cong B^{5}$, so $M \cup \bar{M}^{\prime} \cong S^{4}$.

(2) The doubles $M \cup \bar{M}$ and $M^{\prime} \cup \bar{M}^{\prime}$ are both diffeomorphic to $S^{4}$.

(3) There is a diffeomorphism $\tilde{\theta}: M \rightarrow M^{\prime}$, so that $\left.\tilde{\theta}\right|_{\partial M}$ composed with the gradient flow identification $\partial M^{\prime} \cong \partial M$ is an involution $\theta: \partial M \rightarrow \partial M$ (so $M \cup \bar{M}^{\prime}=M \cup_{\theta} \bar{M}$ ).

Some of this information is summarized in Figure 2

We will explain point 2 above as a warm up to the non simply connected case, Theorem 4.2. We recall that $N$ is built from a neighbourhood $N_{0}=n(A \cup D)$ of the ascending and descending spheres arranged (with additional intersection 


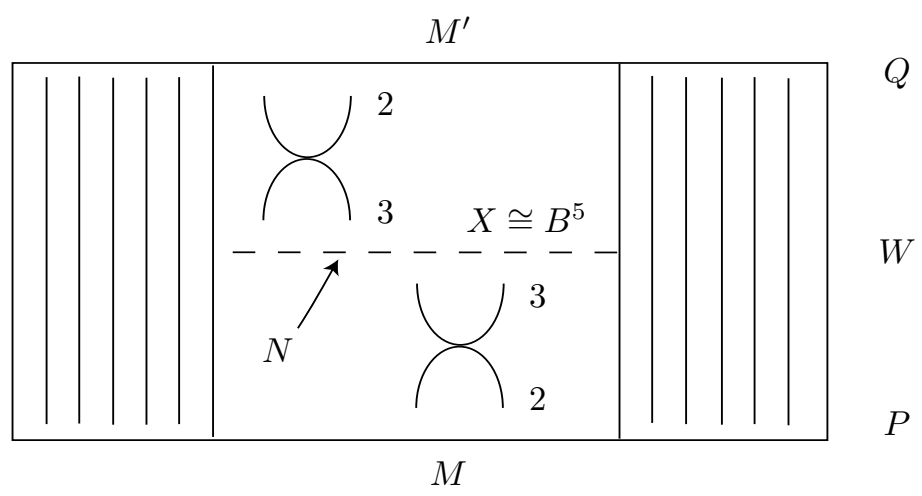

Figure 2: Summary of conditions 1,2,3

points if necessary) so that $\pi_{1}\left(L \backslash N_{0}\right)$ is trivial. A model picture of $N_{0}$ is shown schematically in Figure 3 A more detailed representation, using the Kirby calculus notation (see [3, 11]) is given in Figure 4. More complicated configurations of $A \cup D$ require no new ideas, only more notation, so we will treat the model case. Fix a 4-dimensional handle decomposition $\mathcal{H}$ (without

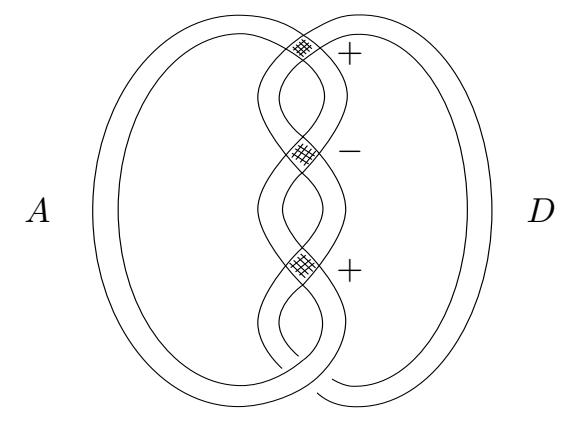

Figure 3: A schematic picture of the neigbourhood $N_{0}$ of $A \cup D$

0 -handles $)$ of $\left(L \backslash N_{0}, \partial N_{0}\right)$. Form $N_{1}:=N_{0} \cup$ all 1-handles of $\left.\mathcal{H}\right\}$ then $N=$ $N_{1} \cup$ (certain 2 -handles). The 2 -handles are made by stabilizing $\mathcal{H}$ with $(2,3)$ handle pairs and passing the new 2 -handles over suitable combinations of 2 handles of $\mathcal{H}$. Since $\pi_{1}\left(L \backslash N_{1}\right)$ is trivial, we have complete freedom in choosing the relations that these new 2 -handles determine in $\pi_{1}\left(\partial\left(L \backslash N_{1}\right)\right)=\pi_{1}\left(\partial N_{1}\right)$. Since $\pi_{1}\left(\partial N_{1}\right) \rightarrow \pi_{1}\left(N_{1}\right)$ is an epimorphism we also have complete freedom to add 2-handle relations to the presentation of $\pi_{1}\left(N_{1}\right)$. We will describe the relations that we introduce shortly. Let $M_{0}:=N_{0} / A, M_{1}:=N_{1} / A, M:=$ $N / A$, and similarly $M_{0}^{\prime}:=N_{0} / D$, etc. Now $M_{0}, M_{1}$ and $M$ result from surgery 


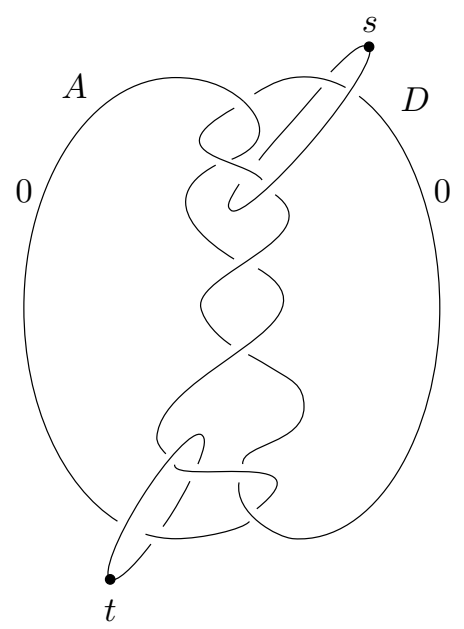

Figure 4: A picture of $N_{0}$ in Kirby calculus notation

on $A$ and we represent this diagrammatically by replacing the the 0 -framing at $A$ with a dot, which indicates that the surgery has converted the 2 -handle into a 1 -handle (see Figure 5). We proceed similarly for $M_{0}^{\prime}, M_{1}^{\prime}$ and $M^{\prime}$.

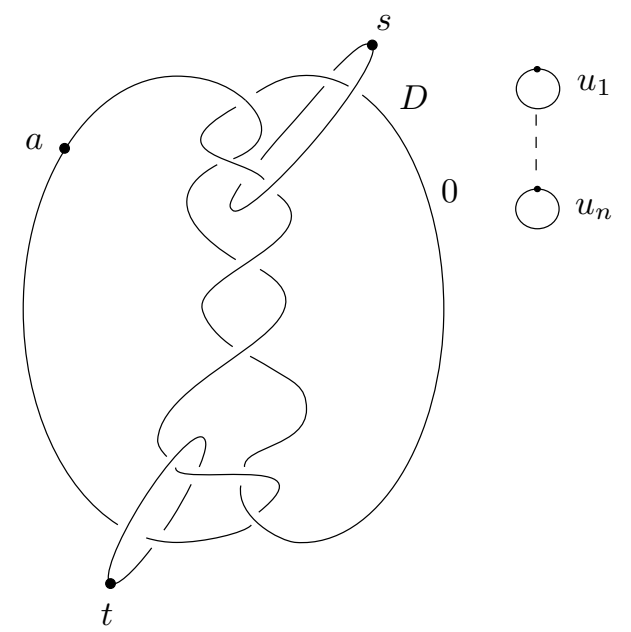

Figure 5: A picture of $M$ in Kirby calculus notation. Surgery on $A$ has converted one of the 2-handles into a 1-handle

To obtain $M \cup \bar{M} \cong S^{4}$, it is sufficient to show that $M \times I \cong B^{5}$, the 5 ball. But $M$ has just been described as a 1,2-handle body, so $M \times I$ is also a 1,2 -handle body and in dimensions $d \geq 5$, only the group theoretic 
presentation $\rho$ is relevant in determining if the handle body is a ball: $M \times I$ is a ball, $B^{5}$, if $\rho$ is "deformable" to, or "Andrews-Curtis related" to, the empty presentation. The presentation $\rho$ that we may read off from Figure 5 has generators $a, s, t, u_{1}, \ldots, u_{n}$ and so far only one relation: $t^{-1} a t a^{-1} s^{-1} a s$. This length 7 relation may not look like a promising start for an a standard presentation of the trivial group, but we can begin by choosing two new 2handles representing $t$ and $s$, which collapse the relation to $a$. From here, simply choose 2 -handles representing length 1 relations $u_{1}, \ldots, u_{n}$. In this way, $N$ is built from $N_{1}$ so that both $M \times I \cong B^{5}$ and $M^{\prime} \times I \cong B^{5}$. For the construction of the involution $\theta$, see lemma 2 in 13 . The statement that $X \cong B^{5}$ in point 1 above can be extracted from our proof of theorem 4.2 in the case $k=0$.

We can now prove the following:

Theorem 4.1 UTQFTs cannot distinguish 1-connected smooth 4-manifolds which are homotopy equivalent. In fact, even a less rigid "theory", where Atiyah's gluing axiom is only enforced along homology 3-spheres, will likewise be unable to distinguish homotopy equivalent 4-manifolds.

Proof It is well known that smooth homotopy equivalent 4 -manifolds $P$ and $Q$ are smoothly $h$-cobordant. In the preceding notation write $P=C \cup_{S} M$ and $Q=C \cup_{S} M^{\prime}$, where $S=\partial M$. Let $Z$ be a UTQFT and let $V(S)$ be the Hilbert space assigned to $S$ by $Z$, so $Z(C), Z(M), Z\left(M^{\prime}\right) \in V(S)$. Now $\left\langle M-M^{\prime}, M-M^{\prime}\right\rangle=S^{4}-S^{4}-S^{4}+S^{4}=0 \in \mathcal{M}^{4}$, so $\left\langle Z\left(M-M^{\prime}\right), Z\left(M-M^{\prime}\right)\right\rangle=$ $0 \in V(S)$ and by unitarity, $Z\left(M-M^{\prime}\right)=0 \in V_{S}$. Hence $Z(M)=Z\left(M^{\prime}\right)$. Finally, $Z(P)=\langle Z(C), Z(M)\rangle=\left\langle Z(C), Z\left(M^{\prime}\right)\right\rangle=Z(Q)$.

We may extend this result to the non-simply connected setting:

Theorem 4.2 UTQFTs cannot distinguish smoothly s-cobordant 4-manifolds.

Remark The proof is a mild generalization of the preceding middle level analysis. It should be noted that the 3-manifolds whose Hilbert spaces we must now consider are no longer homology 3 -spheres, but instead admit maps to $\underset{k}{\vee} S^{1}$ and have vanishing $H_{1}\left(\cdot ; \mathbb{Z}\left[\pi_{1}\left(\underset{k}{\vee} S^{1}\right)\right]\right)$, ie, the corresponding covers have perfect fundamental groups.

Proof of Theorem 4.2 We assume our manifolds are oriented. Again, let $(W ; P, Q)$ be the $s$-cobordism with $0,1,4$ and 5 -handles eliminated. Let $L$, 
again, be the middle level. Find ascending spheres $A=\cup A_{i}$ and descending spheres $D=\cup D_{j}$ for a handle structure exhibiting triviality in the Whitehead group, and so that $\pi_{1}(L \backslash(A \cup D)) \rightarrow \pi_{1}(L)$ is an isomorphism. This may require stabilizations and "finger moves" between the original $A$ and $D$.

The use of finger moves - the inverse Whitney's famous trick [17 — to improve fundamental groups goes back to Casson's constructions [7. The particular incarnation used here is explained in Section 7.1.D of [10], the first half of page 105 being the crux.

Our goal is to engulf $A \cup D$ in $N \subset L$ so that there is a corresponding sub- $s-$ cobordism $\left(X ; M, M^{\prime}\right)$ so that the complement has the gradient product structure and

(1) $X \cong \underset{k}{t}\left(S^{1} \times B^{4}\right)$, so $M \cap \bar{M}^{\prime} \cong \underset{k}{\sharp}\left(S^{1} \times S^{3}\right)$.

(2) The doubles $M \cup \bar{M}$ and $M^{\prime} \cup \bar{M}^{\prime}$ are both diffeomorphic to $\underset{k}{\sharp}\left(S^{1} \times S^{3}\right)$.

By choosing $\Delta$ symmetrically below we may also arrange that $M \cong M^{\prime}$ and that the corresponding $\theta: \partial M \rightarrow \partial M$ is an involution, but these statements are irrelevant to the conclusions on UTQFTs so we do not expatiate.

Since we are trying to build an $N$ with $\pi_{1}(N)$ a large free group, rather than a trivial group, we will not have to be as careful in enlarging $A \cup D$ to $N$ as before. Let $\Delta$ be a union of immersed Whitney disks for $A \cap D$ which pair "excess" double points (see Figure 6). Set $N=n(A \cup D \cup \Delta)$ ). Now $X$ is a thickening of $D_{A}^{3} \cup D_{D}^{3} \cup \Delta$, where $D_{A}^{3}$ and $D_{D}^{3}$ are the 3 -disks descending from $A$ and ascending from $D$, so $X$ manifestly collapses to a wedge of circles. Thus, $X \cong \underset{k}{\natural}\left(S^{1} \times B^{4}\right)$, since $W$ is orientable.

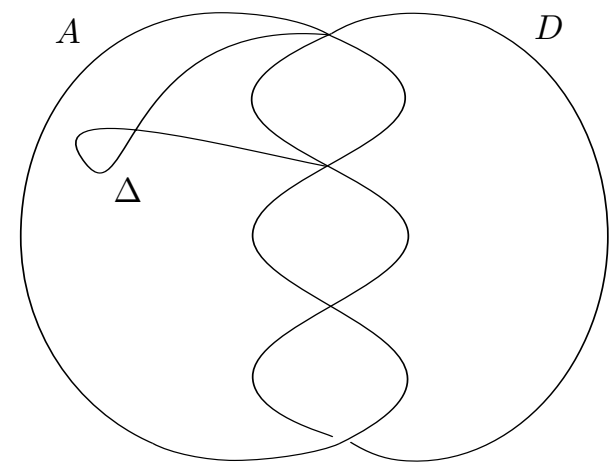

Figure 6: A picture of $A \cup D \cup \Delta$ 
The final point is that $D(M) \cong D\left(M^{\prime}\right)=\underset{k}{\natural} S^{1} \times S^{3}$. As before, this reduces to seeing a 1-handle presentation for $M\left(M^{\prime}\right)$ and verifying that that presentation deforms to the standard presentation of the $k$-generator free group. The model diagram for $M$ is given in Figure 7 .

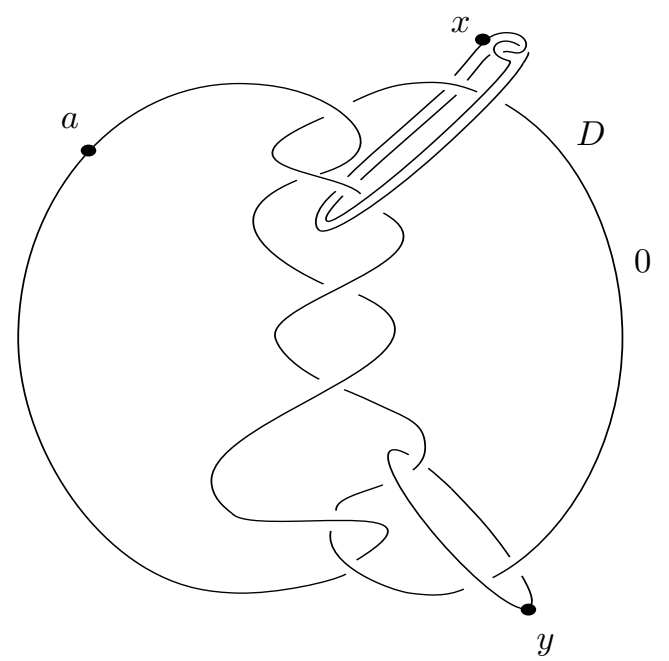

Figure 7: Model diagram for $M$

The reader should compare this with Figure 3, The first thing we notice is that attaching a 3 -handle (which effects a surgery on $A$ ) has been described by removing a zero label and adding a dot, indicating a 1-handle. Locally we are simply creating a bordism from $S^{2} \times D^{2}$ to $D^{3} \times S^{1}\left(\right.$ rel $\left.S^{2} \times S^{1}\right)$ by attaching a 3-handle $\left(D^{3} \times D^{2}, S^{2} \times \frac{1}{2} D^{2}\right)$ to $S^{2} \times \frac{1}{2} D^{2} \subset S^{2} \times D^{2}$. The bottom of the bordism $S^{2} \times D^{2}$ is represented by a trivial 2-handle, the top by a 1-handle.

The corresponding model diagram for $M^{\prime}$ is obtained by replacing the 1 -handle $a$ by a 0 -framed 2-handle $A$ and the 0 -framed 2-handle $D$ by a $1-$ handle $d$. The Whitehead double (curve $x$ in the figure) arises from the attachment of $\Delta$; there will be one such $x$ for each double point on $\Delta$, whereas only a single $y$ curve is present for each pair $\left(A_{i}, D_{i}\right)$. The boundary of the 2-handle core $D$ reads " $a$ ", so the presentation is a standard one for the free group: $\{a, x, y: a\}$. To see this presentation, find disjoint surfaces $\bar{a}, \bar{x}$ and $\bar{y}$ bounding $a, x, y$ such that $D \cap(\bar{a} \cup \bar{x} \cup \bar{y})$ is just one point lying on $\bar{a}$. As $D$ crosses $\bar{a}, \bar{x}$ and $\bar{y}$ it reads its relation in the free group generated by $a, x$ and $y$; the result is simply a smaller free group. The general case, involving additional stabilizations and more double points, is similar. 


\section{Problems}

Problem 1a Given $x \in \mathcal{M}_{S}^{d}$ with $\langle x, x\rangle \neq 0$, is it possible to construct a UTQFT which assigns to $x$ a nonzero vector $\tilde{x} \in V_{S}$ ?

Problem 1b Similarly, given $x \in \mathcal{M}_{S}^{d}$ such that all $d$-dimensional UTQFTs assign the zero vector to $x$, does it follow that $\langle x, x\rangle=0$ ?

Problem 2 Is there a 3 -manifold $S$ and a nonzero vector $x \in \mathcal{M}_{S}^{4}$ such that $\langle x, y\rangle=0$ for all $y \in \mathcal{M}_{S}^{4}$ ? Such an $x$ would be called "singular".

A positive answer to this question would give an example of a combination of 4 -manifolds that will be sent to 0 in any TQFT with a non singular quadratic form, not just in unitary TQFTs. (In a UTQFT $Z,\langle Z(x), Z(x)\rangle=0$ implies that $\langle Z(x), Z(y)\rangle=0$ for all $y$.)

Problem 3 Analyze the 3-dimensional pairings.

Problem 4 Analyze the pairings in dimensions $d \geq 5$.

Problem 5 In dimension $d=4$ characterize the zero locus of the pairing in more detail. For instance, are there any elements in the zero locus of the pairing that have an odd number of manifolds with non-zero coefficients?

Even numbers of nonzero coefficients may be obtained using manifolds which are disjoint unions of the examples given already

Problem 6 Consider the same problem in the piecewise linear and topological categories.

Problem 7 Consider coefficients other than $\mathbb{C}$.

This is almost certainly of interest, because there exist classes of TQFTs whose invariants take values in rings other than $\mathbb{C}$. Clearly the pairings will never be positive in any dimension for coefficient rings with elements $x$ with satisfy $x \bar{x}=0$, but nevertheless, even for such rings, a characterisation of the nullity may be interesting. For example, in the ring $(\mathbb{Z} / 7 \mathbb{Z})[\omega]$ with $\omega=e^{2 \pi i / 7}$ and the involution given by extension of $\overline{1}=1$ and $\bar{\omega}=\omega^{-1}$, there are elements $a$ for which $a \bar{a}=0$, but using the Milnor sphere of order 7 , one can construct more interesting examples $x \in \mathcal{M}_{S^{6}}[(\mathbb{Z} / 7 \mathbb{Z})[\omega]]$ which have $\langle x, x\rangle=0 \in \mathcal{M}[(\mathbb{Z} / 7 \mathbb{Z})[\omega]]$, such as $x=\sum_{i=0}^{6} \omega^{i}\left(B^{6}, \theta_{i}\right)$, where $\theta$ is the "clutching map" for the Milnor sphere. 


\section{References}

[1] M Atiyah, The geometry and physics of knots, Lezioni Lincee. [Lincei Lectures], Cambridge University Press, Cambridge (1990) MathReview

[2] J J Andrews, M L Curtis, Free groups and handlebodies, Proc. Amer. Math. Soc. 16 (1965) 192-195 MathReview

[3] S Akbulut, A fake compact contractible 4-manifold, J. Differential Geom. 33 (1991) 335-356 MathReview

[4] S Akbulut, R C Kirby, Mazur manifolds, Michigan Math. J. 26 (1979) 259284 MathReview

[5] S Akbulut, R C Kirby, A potential smooth counterexample in dimension 4 to the Poincaré conjecture, the Schoenflies conjecture, and the Andrews-Curtis conjecture, Topology 24 (1985) 375-390 MathReview

[6] I Agol, N M Dunfield, P A Storm, W P Thurston, Lower bounds on volumes of hyperbolic Haken 3-manifolds, arXiv:math.DG/0506338

[7] A J Casson, Three lectures on new-infinite constructions in 4-dimensional manifolds, from: "À la recherche de la topologie perdue", Progr. Math. 62, Birkhäuser Boston, Boston, MA (1986) 201-244 MathReview

[8] C L Curtis, M H Freedman, W C Hsiang, R Stong, A decomposition theorem for $h$-cobordant smooth simply-connected compact 4-manifolds, Invent. Math. 123 (1996) 343-348 MathReview

[9] MH Freedman, The topology of four-dimensional manifolds, J. Differential Geom. 17 (1982) 357-453 MathReview

[10] M H Freedman, F Quinn, Topology of 4-manifolds, Princeton Mathematical Series 39, Princeton University Press, Princeton, NJ (1990) MathReview

[11] R C Kirby, The topology of 4-manifolds, Lecture Notes in Mathematics 1374, Springer-Verlag, Berlin (1989) MathReview

[12] R C Kirby, Akbulut's corks and h-cobordisms of smooth, simply connected 4-manifolds, Turkish J. Math. 20 (1996) 85-93 MathReview

[13] R Matveyev, A decomposition of smooth simply-connected $h$-cobordant 4 manifolds, J. Differential Geom. 44 (1996) 571-582 MathReview

[14] J Milnor, A unique decomposition theorem for 3-manifolds, Amer. J. Math. 84 (1962) 1-7 MathReview

[15] H Schubert, Die eindeutige Zerlegbarkeit eines Knotens in Primknoten, S.-B. Heidelberger Akad. Wiss. Math.-Nat. Kl. 1949 (1949) 57-104 MathReview

[16] F Waldhausen, Heegaard-Zerlegungen der 3-Sphäre, Topology 7 (1968) 195203 MathReview

[17] H Whitney, The self-intersections of a smooth $n$-manifold in $2 n$-space, Ann. of Math. (2) 45 (1944) 220-246 MathReview 